TITLE:

\title{
Development of low noise cantilever deflection sensor for multienvironment frequency- modulation atomic force microscopy
}

\section{$\operatorname{AUTHOR}(\mathrm{S}):$}

Fukuma, Takeshi; Kimura, Masayuki; Kobayashi, Kei; Matsushige, Kazumi; Yamada, Hirofumi

\section{CITATION:}

Fukuma, Takeshi ...[et al]. Development of low noise cantilever deflection sensor for multienvironment frequency-modulation atomic force microscopy. REVIEW OF SCIENTIFIC INSTRUMENTS 2005, 76(5): 053704.

\section{ISSUE DATE:}

2005-05

URL:

http://hdl.handle.net/2433/39812

\section{RIGHT:}

Copyright 2005 American Institute of Physics. This article may be downloaded for personal use only. Any other use requires prior permission of the author and the American Institute of Physics. 


\title{
Development of low noise cantilever deflection sensor for multienvironment frequency-modulation atomic force microscopy
}

\author{
Takeshi Fukuma and Masayuki Kimura \\ Department of Electronic Science and Engineering, Kyoto University, Kyoto 615-8510, Japan \\ Kei Kobayashi \\ International Innovation Center, Kyoto University, Kyoto 606-8501, Japan \\ Kazumi Matsushige and Hirofumi Yamada ${ }^{\text {a) }}$ \\ Department of Electronic Science and Engineering, Kyoto University, Kyoto 615-8510, Japan
}

(Received 7 January 2005; accepted 13 February 2005; published online 15 April 2005)

\begin{abstract}
We have developed a low noise cantilever deflection sensor with a deflection noise density of $17 \mathrm{fm} / \sqrt{\mathrm{Hz}}$ by optimizing the parameters used in optical beam deflection (OBD) method. Using this sensor, we have developed a multienvironment frequency-modulation atomic force microscope (FM-AFM) that can achieve true molecular resolution in various environments such as in moderate vacuum, air, and liquid. The low noise characteristic of the deflection sensor makes it possible to obtain a maximum frequency sensitivity limited by the thermal Brownian motion of the cantilever in every environment. In this paper, the major noise sources in OBD method are discussed in both theoretical and experimental aspects. The excellent noise performance of the deflection sensor is demonstrated in deflection and frequency measurements. True molecular-resolution FM-AFM images of a polydiacetylene single crystal taken in vacuum, air, and water are presented. (C) 2005 American Institute of Physics. [DOI: 10.1063/1.1896938]
\end{abstract}

\section{INTRODUCTION}

True atomic and molecular resolution of frequencymodulation atomic force microscopy (FM-AFM) (Ref. 1) has been demonstrated on various surfaces such as semiconductors, ${ }^{2,3}$ metals, ${ }^{4,5}$ alkali halides, ${ }^{6}$ and organic materials. ${ }^{7}$ However, all the high-resolution FM-AFM images presented so far were obtained in ultrahigh vacuum (UHV) environments, which has prevented many practical applications in air and liquid. In particular, high-resolution imaging in liquid is essential for investigating biological samples such as DNA, proteins and living cells in their physiological environments.

The major difficulty in high-resolution imaging in air and liquid is a low $Q$ factor of the cantilever resonance. In FM-AFM, the frequency noise arising from the cantilever thermal Brownian motion increases as the $Q$ factor decreases. ${ }^{1}$ In addition, the low $Q$ factor results in a wide spectral width of the cantilever self-oscillation, leading to a large frequency noise in the frequency-modulation (FM) detection. Due to these problems, high-resolution FM-AFM imaging in low $Q$ environments (i.e., in air and liquid) has been regarded as a challenging target.

However, detailed noise analysis of frequency measurements in FM-AFM, which will be presented in this article, revealed that the force sensitivity of the conventional FMAFM is limited not only by the cantilever thermal Brownian motion, namely, $Q$ factor but also by the noises arising from the cantilever deflection sensor. Thus, in order to achieve " $Q$

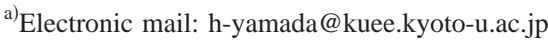

limited noise performance" in FM-AFM, the frequency noise caused by the deflection sensor must be reduced less than that by the thermal Brownian motion.

Since the invention of $\mathrm{AFM}^{8}{ }^{8}$ a wide variety of cantilever deflection sensors have been used. ${ }^{8-15}$ Among them, the highest deflection sensitivities have been achieved with optical interferometers. For example, Rugar et al. ${ }^{16}$ reported a fiberoptic interferometer with a deflection noise density of $17 \mathrm{fm} / \sqrt{\mathrm{Hz}}$. Schönenberger et al. ${ }^{12}$ showed a polarizing optical interferometer with a deflection noise density of $6 \mathrm{fm} / \sqrt{\mathrm{Hz}}$. In spite of those excellent deflection sensitivities, the interferometers have not been used in commercially available AFMs. Instead, most of the commercially available AFMs utilize optical beam deflection (OBD) method ${ }^{11}$ because of the simple experimental setup and the easy optical beam alignment. Although theoretical studies suggested that the theoretical limits of the deflection sensitivities obtained by these two methods are nearly the same, ${ }^{17}$ the deflection noise densities in the practical OBD sensors (typically $100-1000 \mathrm{fm} / \sqrt{\mathrm{Hz}}$ ) have been worse than those in the optical interferometers.

In this study, we have investigated the major factors that limit the deflection sensitivity of the actual OBD sensor both in theoretical and experimental aspects. Then, we have developed a low noise OBD sensor with a deflection noise density of $17 \mathrm{fm} / \sqrt{\mathrm{Hz}}$. Using this sensor, we have developed a multienvironment FM-AFM that can achieve true molecular resolution in various environments such as in vacuum, air, and liquid. The noise performance of the sensor was quantitatively investigated in the deflection and frequency mea- 

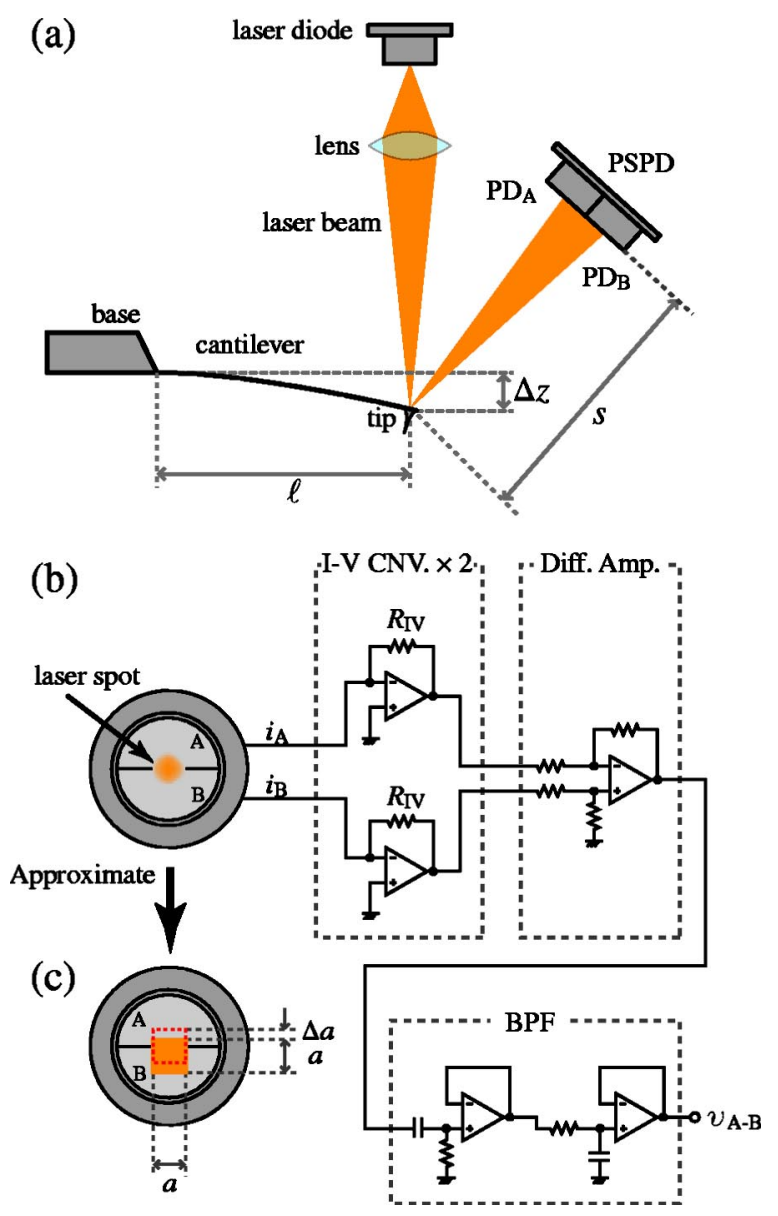

FIG. 1. (Color online) (a) Typical experimental setup for the cantilever deflection sensor using the OBD method. (b) The PSPD and the following electronic circuits including $I-V$ converters, a differential amplifier and a band pass filter. (c) An approximated laser spot on the PSPD.

surements. True molecular-resolution FM-AFM imaging was performed on a polydiacetylene single crystal in various environments. ${ }^{18,19}$

\section{MAJOR NOISE SOURCES IN OBD METHOD}

\section{A. Basic principle}

Figure 1(a) shows a typical experimental setup for a cantilever deflection sensor using OBD method. In OBD method, a focused laser beam is irradiated onto the backside of the cantilever. The bounced laser beam is detected with a position sensitive photodetector (PSPD) which consists of two adjacent photodiodes $\left(\mathrm{PD}_{\mathrm{A}}\right.$ and $\left.\mathrm{PD}_{\mathrm{B}}\right)$. The laser beam is aligned so that the laser spot on the PSPD is positioned at its center [Fig. 1(b)]. The cantilever displacement $\Delta z$ induces the cantilever deflection $\Delta \theta$ which is given by ${ }^{20}$

$$
\Delta \theta=\frac{3}{2} \frac{\Delta z}{\ell},
$$

where $\ell$ is the cantilever length. This cantilever deflection gives rise to the laser beam deflection of $2 \Delta \theta$. Thus, the laser spot irradiated onto the PSPD is displaced by $\Delta a$ which is given by

$$
\Delta a=3 \frac{s}{\ell} \Delta z
$$

where $s$ is the distance between the tip and the PSPD. The amplification factor $\beta$ given by $\beta=\Delta a / \Delta z$ is typically about $10^{3}$. Owing to the large amplification factor, the OBD method can achieve a high deflection sensitivity.

The photo-induced currents $\left(i_{\mathrm{A}}\right.$ and $\left.i_{\mathrm{B}}\right)$ from the two photodiodes are independently detected with $I-V$ converters. The detected signals are fed into a differential amplifier which produces the voltage signal $\left(v_{\mathrm{A}-\mathrm{B}}\right)$ proportional to the current difference $\left(i_{\mathrm{A}-\mathrm{B}}\right)$ between $i_{\mathrm{A}}$ and $i_{\mathrm{B}}$. Then, the unwanted frequency components are eliminated with a band pass filter (BPF) having a center frequency near the cantilever resonance. The displacement of the laser spot causes one of the photodiodes to collect more light than the other, leading to the deviation $\left(\Delta i_{\mathrm{A}-\mathrm{B}}\right)$ in $i_{\mathrm{A}-\mathrm{B}}$.

In dynamic-mode AFM, the cantilever displacement does not occur in a static manner but dynamically. On the other hand, the time response of the photodetector, including the PSPD and the preamplifier, is limited by the influence of the junction capacitance of a photodiode. Thus, with a frequency-dependent gain reduction factor $\left(\xi_{\mathrm{PD}}\right)$ taken into account, the variation of the cantilever deflection signal $\left(\Delta v_{\mathrm{A}-\mathrm{B}}\right)$ is given by

$$
\Delta v_{A-B}=\eta \alpha P \xi_{\mathrm{PD}} R_{\mathrm{IV}} \frac{3 s}{\ell a} \Delta z,
$$

where $R_{\mathrm{IV}}, \eta, \alpha$, and $P$ are the feedback resistor of the photodiode preamplifier, the efficiency of the light-to-current conversion at the photodiodes, the laser power attenuation coefficient in the optical path, and the output power of the laser diode, respectively. Note that the shape of the laser spot is assumed to be square with a dimension of $a \times a$ as shown in Fig. 1(c).

\section{B. Major noise sources}

One of the major noise sources in OBD method is the photodiode shot noise. The voltage noise arising from the shot noise $\left(\delta v_{s}\right)$ is given by

$$
\delta v_{s}=\xi_{\mathrm{PD}} R_{\mathrm{IV}} \sqrt{2 e \eta \alpha P B_{\mathrm{BPF}}},
$$

where $e$ and $B_{\mathrm{BPF}}$ are the elementary charge and the bandwidth of the BPF. From Eqs. (3) and (4), the effective cantilever deflection noise density caused by the shot noise is given by

$$
n_{z s}=\frac{\delta v_{s} / \sqrt{B_{\mathrm{BPF}}}}{\Delta v_{\mathrm{A}-\mathrm{B}} / \Delta z}=\frac{\ell a}{3 s} \sqrt{\frac{2 e}{\eta \alpha P}} .
$$

This equation shows that $n_{z s}$ is reduced by increasing $P$. In most of the well-designed OBD sensors, $n_{z s}$ is the predominant noise component.

Another major noise source in OBD method is the Johnson noise originated from the resistor $\left(R_{\mathrm{IV}}\right)$ used in the $I-V$ converters. The voltage noise arising from the Johnson noise is given by 


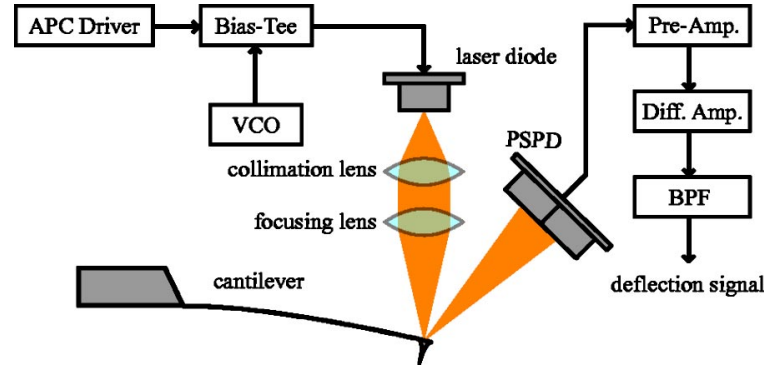

FIG. 2. (Color online) Schematic drawing of the experimental setup for our low noise cantilever deflection sensor using OBD method.

$$
\delta v_{J}=\sqrt{4 k_{B} T N_{\mathrm{PD}} R_{\mathrm{IV}} B_{\mathrm{BPF}}},
$$

where $N_{\mathrm{PD}}, k_{B}$, and $T$ are the number of photodiodes used in the PSPD, Boltzmann constant and temperature, respectively. Although a two-segment photodiode is used as the PSPD in the setup shown in Fig. 1, a four-segment photodiode is also often used for detecting both vertical and lateral cantilever deflections. Thus, $N_{\mathrm{PD}}$ is 2 or 4 in most cases. From Eqs. (3) and (6), the effective cantilever deflection noise density caused by the Johnson noise $\left(n_{z J}\right)$ is given by

$$
n_{z J}=\frac{\delta v_{J} / \sqrt{B_{\mathrm{BPF}}}}{\Delta v_{\mathrm{A}-\mathrm{B}} / \Delta z}=\frac{\ell a}{3 s} \frac{1}{\eta \alpha P \xi_{\mathrm{PD}}} \sqrt{\frac{4 k_{B} T N_{\mathrm{PD}}}{R_{\mathrm{IV}}}} .
$$

This equation shows that $n_{z J}$ reduces with increasing $P$ and $R_{\mathrm{IV}}$. In most of the OBD sensors, $n_{z J}$ is smaller than $n_{z s}$. However, $n_{z J}$ increases with decreasing $\xi_{\mathrm{PD}}$ while $n_{z s}$ remains constant. Thus, $n_{z J}$ may exceed $n_{z s}$ for high frequency cantilevers due to the insufficient bandwidth of the preamplifier.

In general, the deflection noise arising from the intensity fluctuation of the laser diode driven by an automatic power control (APC) circuit is negligible compared to those from the photodiode shot noise and the load resistance Johnson noise. This is because noise arising from the laser intensity fluctuation is mostly eliminated as a common mode noise at the differential amplifier if its common mode rejection ratio (CMRR) at the cantilever vibration frequency is sufficiently large. However, even when the amount of the laser intensity fluctuation is negligible, only small amount of the mode fluctuations in the laser diode can induce a drastic increase of the deflection noise. This is because the laser mode fluctuation causes a fluctuation of the spatial distribution of the laser spot on the PSPD. Such a fluctuation of the laser spot pattern produces a differential mode noise which is further magnified by a differential amplifier. Since the behavior of this noise component is strongly dependent on the characteristics of the laser diodes and other optical components used in OBD method, its quantitative estimation is very difficult. Thus, we discuss this issue from more practical aspects in the following section.

\section{LOW NOISE CANTILEVER DEFLECTION SENSOR}

Figure 2 schematically shows the experimental setup for our low noise cantilever deflection sensor. Although the design is basically the same as those used in conventional OBD sensors, our sensor has a much lower deflection noise density than those produced by the conventional OBD sensors. In the

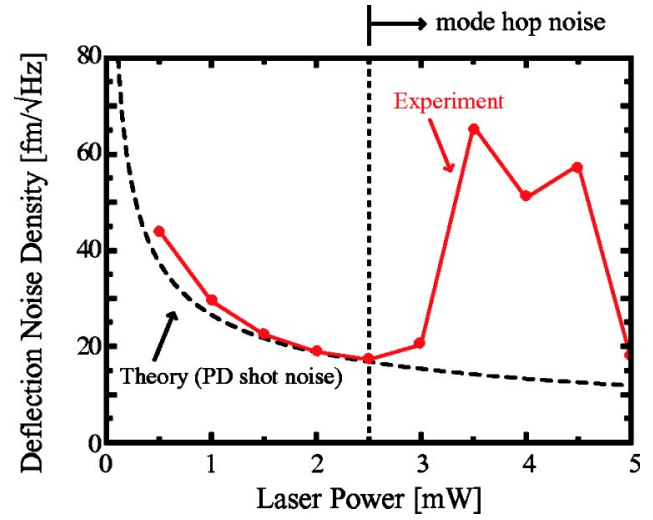

FIG. 3. (Color online) Laser power dependence of the cantilever deflection noise density measured with our deflection sensor in air. The solid circles indicate the experimentally measured deflection noise densities while the dotted line shows the theoretically calculated values of $n_{z s}$ using Eq. (5). The parameters used in the calculation are given by $\ell=100 \mu \mathrm{m}, s=5 \mathrm{~cm}, \eta$ $=0.5 \mathrm{~A} / \mathrm{W}, a=1 \mathrm{~mm}$, and $\alpha=0.4$. The laser power was modulated with a frequency of $300 \mathrm{MHz}$ to suppress the optical feedback noise and the optical interference noise. A Si cantilever with an Al backside coating (Nanosensors: NCHR) was used.

following subsections, distinctive features of our sensor required for the low noise characteristic are described in detail.

\section{A. Optimal laser output power}

An index-guided laser diode (Hitachi: HL6312G) with a rated output power of $5 \mathrm{~mW}$ and a nominal output wavelength of $635 \mathrm{~nm}$ is used in our sensor. The averaged output power of the laser diode is maintained constant using an APC driver (ThorLabs: IP500). Figure 3 shows laser power dependence of the deflection noise density $\left(n_{z}\right)$ measured with our deflection sensor in air. In this figure, the solid circles show experimentally measured values while the dotted line shows theoretically calculated values of $n_{z s}$ with Eq. (5). The relevant parameters used in the calculation are given in the figure caption of Fig. 3. When the laser power is less than $2.5 \mathrm{~mW}$, the experimentally measured values show good agreement with the theoretically calculated values. This indicates that the noise performance of the sensor is basically limited by the photodiode shot noise. Thus, the deflection noise density decreases with increasing the laser power. However, the deflection noise density drastically increases as the laser power exceeds $2.5 \mathrm{~mW}$. The result suggests the contribution from the laser mode hop noise in the high output power regime.

In general, a laser diode operated with a relatively small output power compared to its rating shows intensity fluctuations originated from the spontaneous light emission. This noise is referred to as "quantum noise." As the laser power increases, the quantum noise becomes negligible compared to the averaged laser power. Instead, the intensity fluctuations arising from the hopping of the laser oscillation mode becomes evident in the high output power regime. This noise is referred to as "mode hop noise." The laser mode hopping causes a fluctuation of the laser spot pattern on the PSPD. This leads to a large deflection noise as mentioned in the previous section. The magnitude of the mode hopping does not show a monotonic dependence on the output laser power 
but a oscillatory dependence as seen in Fig. 3. However, the use of the laser power corresponding to the local minimum found in the oscillatory dependence is unrealistic because this laser power often varies depending on the surrounding temperature. Thus, the laser power should not exceed the threshold for the onset of the laser mode hopping. In our setup, since the threshold value varies depending on the temperature from $2 \mathrm{~mW}$ to $3 \mathrm{~mW}$, we adopted the operating laser power of $2 \mathrm{~mW}$.

\section{B. rf laser power modulation}

Besides the intrinsic noises of the laser diode, the quantum noise and the mode hop noise, there are two other noise sources related to the laser beam. The reflection and scattering of the laser beam occasionally produce some part of the laser beam going back into the optical resonator of the laser diode. This optical feedback causes another laser oscillation mode, leading to the increase of the mode hopping. This noise is referred to as "optical feedback noise." On the other hand, some of the reflected or scattered laser beam incidents on the PSPD. This laser beam interferes with other laser beams which come through the different optical paths. Such unexpected optical paths are unstable because of the temperature variations and mechanical drifts. Thus, the laser spot pattern on the PSPD shows fluctuation in a relatively long time scale. This noise is referred to as "optical interference noise." These noises are particularly dominant in liquidenvironment AFMs. In the setup for liquid-environment AFMs, many obstacles such as cover glass and solution are inserted into the optical path of the laser beam. These obstacles induce the reflection and scattering of the laser beam, which increases the optical feedback noise and the optical interference noise.

These optical noises can be suppressed by modulating the laser power with a radio frequency (rf) signal whose frequency is typically $300-500 \mathrm{MHz}^{21,22}$ The rf laser power modulation changes the laser oscillation mode from singlemode to multimode. Since the mode hopping takes place due to the competition among the possible laser oscillation modes in the optical resonator, the multimode laser oscillation is much more insensitive to the optical feedback than the single-mode laser oscillation. Consequently, the rf modulation considerably reduces the mode hopping induced by the optical feedback. In addition, the multimode laser beam has a lower coherence than the single-mode does. Thus, the rf modulation also works well to suppress the optical interference noise.

We used this technique in our deflection sensor. The rf signal is generated with a voltage-controlled oscillator (VCO) (Mini-Circuits: POS-400). The frequency and the magnitude of the rf signal were about $300 \mathrm{MHz}$ and $+9 \mathrm{dBm}$, respectively. The rf signal was added to the current signal produced by the APC driver using a bias-tee (MiniCircuits: PBTC-1GW). Figure 4 shows a waveform of the frequency shift signal measured in water before and after turning off the rf laser power modulation. The waveform shows that the magnitude of the frequency noise suddenly increased after turning off the rf modulation. This demonstrates that the rf modulation is effective to reduce the optical

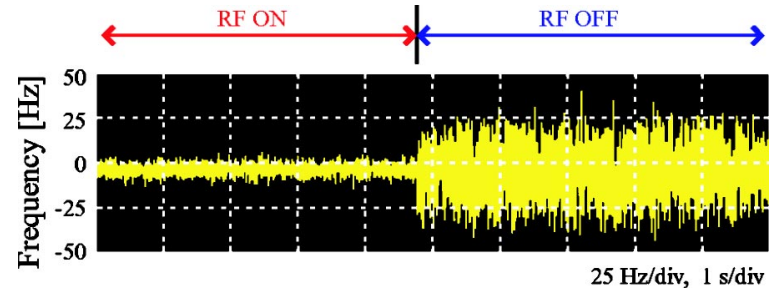

FIG. 4. (Color online) Waveform of the frequency shift signal measured in water before and after turning off the rf laser power modulation $(A=5 \mathrm{~nm}$, $\left.f_{0}=140 \mathrm{kHz}, k=42 \mathrm{~N} / \mathrm{m}, Q=30\right)$. A Si cantilever with an Al backside coating (Nanosensors: NCHR) was used. The cantilever was oscillated in constant amplitude mode.

feedback noise and the optical interference noise. Although the $\mathrm{rf}$ modulation also reduces the intrinsic mode hop noise, it was not completely suppressed in our case. In fact, the laser power dependence shown in Fig. 3 clearly shows the contribution from the intrinsic mod hop noise in spite of the rf laser power modulation. Thus, the averaged laser power should be set at a value below the threshold for the onset of the mode hop noise even with the rf laser power modulation.

\section{Efficiency of optical transmission}

The laser power should be increased for reducing $n_{z s}$ while the maximum laser power is limited by the threshold for the onset of the laser mode hopping. Accordingly, the laser power loss in the optical path from the laser diode to the PSPD should be minimized. This is also clear from Eq. (5) showing that $n_{z s}$ decreases with increasing $\alpha$.

In our deflection sensor, an aspheric collimation and focusing lens pair (ThorLabs: LT230260P) is used for the efficient optical transmission of the laser beam. The collimation lens should have a large numerical aperture (NA) to collect as much as light emitted from the laser diode chip which diverse rapidly with its travel. The NA and the focal length of the collimation lens are 0.55 and $4.5 \mathrm{~mm}$, respectively. The large NA of this collimation lens allows us to collect almost 100\% light emitted from the laser diode. On the other hand, the focusing lens should have a relatively small NA for minimizing the beam divergence in the optical travel from the cantilever backside to the PSPD. The NA and the focal length of the focusing lens are 0.16 and $15.36 \mathrm{~mm}$, respectively. The small NA may limit the minimum laser spot size on the cantilever backside. If the laser spot size exceeds the cantilever width, some of the laser beam will incident on the sample. This may lead to unwanted influence on the sample properties as well as a low $\alpha$ value. The laser spot size on the cantilever backside in our sensor is about $30 \mu \mathrm{m}$ which is almost the same as the width of standard silicon cantilevers. Since the cantilever backside works as an optical mirror in OBD method, the increase of its reflectivity will enhance the efficiency of the optical transmission. In this respect, metal coating of the cantilever backside is beneficial. For example, the reflectivity of the backside of a standard silicon cantilever (Nanosensors: $\mathrm{NCH}$ ) is about 25\% while that of $\mathrm{Al}$ coated one (Nanosensors: NCHR) is about $65 \%$ in our setup. Thus, a metal coated cantilever usually gives a better noise characteristic than uncoated one. 

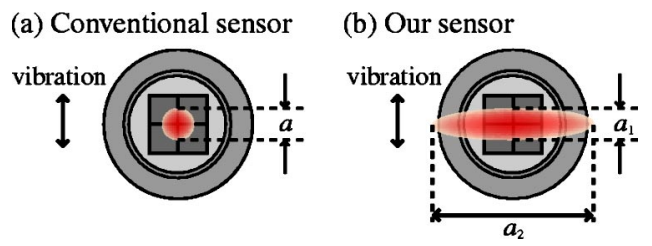

FIG. 5. (Color online) Laser spot pattern on the PSPD in (a) conventional deflection sensor and (b) our sensor. The laser spot size along the short and long axes are $a_{1}=1 \mathrm{~mm}$ and $a_{2}=4 \mathrm{~mm}$, respectively.

The far field pattern of an optical beam emitted from a laser diode usually shows an elliptic shape. This is because the diffraction angle along the $p n$-junction layer is smaller than that perpendicular to the layer. However, the laser spot pattern on the PSPD in conventional deflection sensors shows a nearly round shape as shown in Fig. 5(a). In those sensors, a collimation lens with a low NA partially block off the laser beam. This provides a round shape laser beam at the expense of some laser power. Although this setup allows us to detect cantilever deflections in both lateral and vertical directions, the laser power loss leads to a low deflection sensitivity. On the other hand, the laser spot on the PSPD in our sensor shows an elliptic pattern $\left[a_{1} \times a_{2}\right.$ as shown in Fig. 5 (b) ] because all the laser beam is collected with a collimation lens having a large NA. The rotation angle of the laser beam is adjusted such that the short axis of the elliptic pattern is oriented along the direction of the laser spot displacement. Since $a_{2}$ is longer than the size of the PSPD, about $40 \%$ of the light is lost here. Consequently, the total laser power attenuation $\alpha$ is 0.4 for a metal coated cantilever and 0.15 for a standard silicon cantilever. The effective laser spot size equals to $a_{1}$ since the displacement takes place along this direction. This small effective laser spot size provides an excellent sensitivity to the laser spot displacement.

\section{Wideband photodetector}

The photo-induced current from the PSPD is converted to the voltage signal with a pre-amplifier. The photodetector must have enough bandwidth $\left(B_{\mathrm{PD}}\right)$ for detecting the cantilever vibration. Otherwise, the decrease of $\xi_{\mathrm{PD}}$ results in the increase of $n_{z J}$. Although $n_{z s}$ is the predominant noise source in most of the OBD sensors, $n_{z J}$ may exceed it due to the small $\xi_{\mathrm{PD}}$. From Eqs. (5) and (7), the condition for $n_{z s}<n_{z J}$ is given by

$$
\xi_{\mathrm{PD}}>\sqrt{\frac{2 k_{B} T N_{\mathrm{PD}}}{e \eta \alpha P R_{\mathrm{IV}}}} .
$$

In our case, the condition is $\xi_{\mathrm{PD}}>0.23\left(N_{\mathrm{PD}}=4, \eta=0.5, P\right.$ $=2 \mathrm{~mW}, \alpha=0.4, R_{\mathrm{IV}}=10 \mathrm{k} \Omega$ ).

The resonance frequencies of conventional cantilevers used in FM-AFM are in the range of $100-400 \mathrm{kHz}$. Thus, $B_{\mathrm{PD}}$ of the conventional deflection sensor is mostly less than $600 \mathrm{kHz}$. However, the use of high frequency cantilevers in FM-AFM has recently attracted much attention because it can enhance the force sensitivity and time response. Thus, it is expected that the cantilever resonance frequency used in

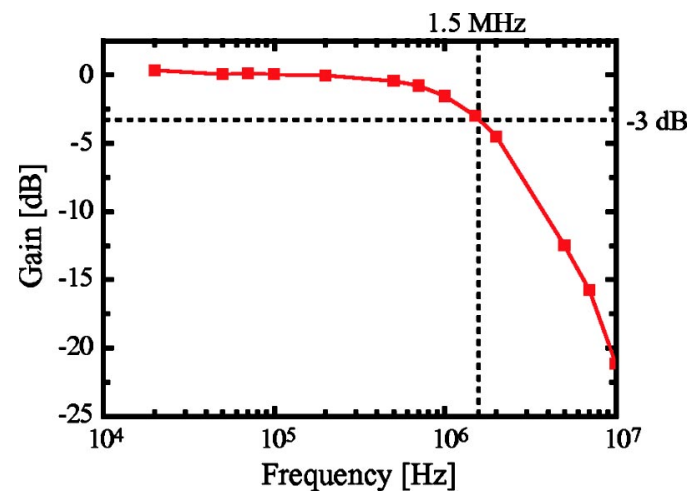

FIG. 6. (Color online) The frequency dependence of the normalized gain of the photodetector including the PSPD and the preamplifier. The gain values are normalized with respect to the dc gain.

FM-AFM will be increased in the near future. The wideband cantilever deflection sensor is essential for detecting the vibration of such high frequency cantilevers.

The bandwidth of the photodetector is mainly determined by the junction capacitance of the photodiode and the gain bandwidth product of the operational amplifier and the feedback resistor used in the preamplifier. In our sensor, a four-segment Si PIN photodiode (Moririca: MI-33H-4D) is used as the PSPD. The total chip size of the PSPD is $2.6 \mathrm{~mm} \times 2.6 \mathrm{~mm}$. The four segments are separated by $50 \mu \mathrm{m}$. Since the PSPD consists of PIN photodiodes, it has a small junction capacitance of $10 \mathrm{pF}$. The preamplifier consists of a quad operational amplifier (Texas Instruments: OPA4354AIDR) and a feedback resistor of $10 \mathrm{k} \Omega$. The operational amplifier has a high gain bandwidth product of $100 \mathrm{MHz}$. Figure 6 shows the frequency dependence of the normalized gain of the photodetector including the PSPD and the preamplifier. The figure shows the photodetector has a $-3 \mathrm{~dB}$ bandwidth of $1.5 \mathrm{MHz}$. This value is much higher than that in conventional deflection sensors.

The voltage signal from the photodetector is fed into a differential amplifier. In OBD method, this differential amplifier is used for eliminating the common mode noise as well as for magnifying the differential mode signal induced by the cantilever vibration. Thus, the differential amplifier should have a high CMRR as well as high differential gain. In addition, the bandwidth of the differential amplifier $\left(B_{\text {dif }}\right)$ should be higher than $B_{\mathrm{PD}}$ to avoid the signal attenuation. In our sensor, the differential amplifier consists of an instrumentation amplifier (Texas Instruments: INA111). The differential gain of this amplifier was set at $20 \mathrm{~dB}$ by using an external resistor of $5.6 \mathrm{k} \Omega$. With this gain, $B_{\text {dif }}$ of the amplifier was about $1.6 \mathrm{MHz}$, which is higher than $B_{\mathrm{PD}}$ of our sensor. The CMRR at $300 \mathrm{kHz}$, which is a typical frequency of the cantilever resonance used in FM-AFM, was about $45 \mathrm{~dB}$. This high CMRR eliminates the common mode noises such as laser intensity fluctuations.

\section{E. Variable frequency band pass filter}

In FM-AFM, the cantilever is usually oscillated with a piezoelectric actuator placed near the cantilever base. In liquid-environments, the vibration of the actuator is transmitted through the surrounding liquid as well as through the 


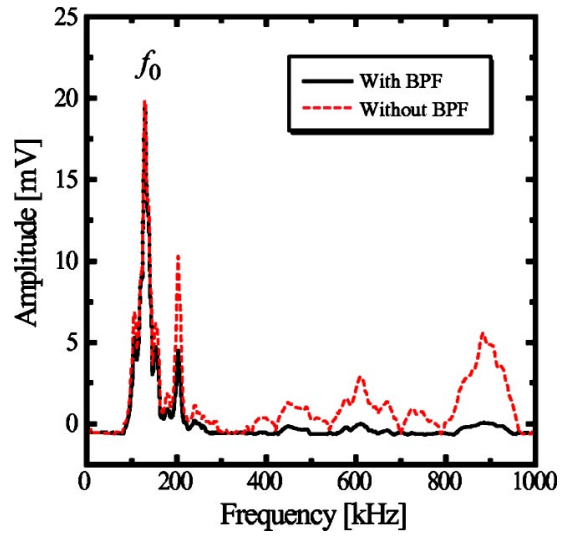

FIG. 7. (Color online) The amplitude response of the cantilever measured by sweeping the frequency of the excitation signal in water. The solid and dotted lines were measured with and without BPF, respectively. A Si cantilever with an Al backside coating (Nanosensors: NCHR) was used.

cantilever base. Thus, the amplitude response of the cantilever vibration shows many unwanted resonances in addition to the fundamental resonance of the cantilever. These resonances prevent a stable cantilever self-oscillation. The selfoscillation frequency often tracks at the unwanted resonances and jumps from one to another.

In our setup, BPF (NF: DT-208D) is used for eliminating the unwanted frequency components contained in the deflection signal. Since the cantilever resonance frequency varies from one to another, the center frequency of the BPF should be adjustable. For DT-208D, it can be changed from $10 \mathrm{kHz}$ to $1.59 \mathrm{MHz}$ with external binary-coded-decimal (BCD) switches. The $Q$ factor of the BPF $\left(Q_{\mathrm{BPF}}\right)$ should be smaller than that of the cantilever resonance to avoid the influence on the phase-frequency characteristic of the cantilever resonance. In our case, $Q_{\mathrm{BPF}}$ was set at 2 which is much smaller than the $Q$ factor of the cantilevers used in our experiments (typically $Q=20-30$ in liquid).

Figure 7 shows the amplitude response of the cantilever measured by sweeping the frequency of the excitation signal in water. The solid and dotted lines were measured with and without the BPF, respectively. A number of unwanted resonance peaks are found in the spectrum taken without BPF while they are well suppressed by the BPF in the other case. The result shows that the BPF works well to suppress the unwanted frequency components in the deflection signal. The BPF is essential for the stable self-oscillation of the cantilever in liquid environments.

\section{MULTIENVIRONMENT FM-AFM}

Using the low noise deflection sensor, we have developed a multienvironment FM-AFM that can achieve true molecular resolution in various environments such as in vacuum, air and liquids. The FM-AFM was developed by modifying a commercially available AFM (JEOL: JSPM4200). The original AFM control electronics was replaced with our homebuilt AFM controller. The original cantilever deflection sensor was replaced with our newly developed OBD sensor. A homebuilt FM detector using a phase-locked loop circuit ${ }^{23}$ was used for the frequency detection.
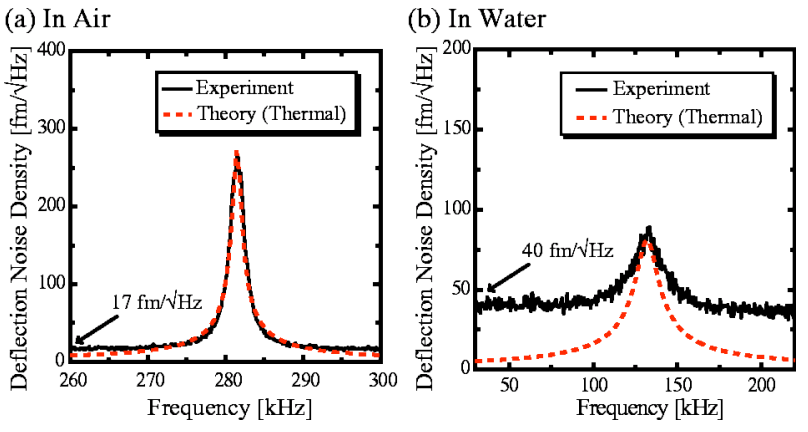

FIG. 8. (Color online) Frequency spectra of cantilever Brownian motion measured in (a) air $\left(Q=241, f_{0}=281.5 \mathrm{kHz}\right)$ and (b) water $\left(Q=10, f_{0}\right.$ $=132 \mathrm{kHz}$ ). The solid lines show experimentally measured values while the dotted lines show theoretically calculated values with Eq. (9). A Si cantilever with an Al backside coating (Nanosensors: NCHR) was used ( $k$ $=30 \mathrm{~N} / \mathrm{m}$ )

\section{A. Measurements of cantilever Brownian motion}

The frequency spectra of the cantilever Brownian vibration induced by the thermal energy is given by ${ }^{1}$

$$
n_{z B}=\sqrt{\frac{2 k_{B} T}{\pi f_{0} k Q} \frac{1}{\left[1-\left(f / f_{0}\right)^{2}\right]^{2}+\left[f /\left(f_{0} Q\right)\right]^{2}}},
$$

where $f, f_{0}$, and $k$ are vibration frequency, resonance frequency, and spring constant of a cantilever, respectively. Figures 8(a) and 8(b) show the frequency spectra of cantilever Brownian motion measured in air and water, respectively. The solid lines show experimentally measured values while the dotted lines show theoretically calculated values with Eq. (9). The peaks found in the spectra correspond to the Brownian vibration at the cantilever resonance while the background white noise comes from the deflection sensor noise. The results reveal that the deflection noise densities arising from our deflection sensor were $17 \mathrm{fm} / \sqrt{\mathrm{Hz}}$ in air and $40 \mathrm{fm} / \sqrt{\mathrm{Hz}}$ in liquid. These values are much smaller than the deflection noise densities obtained with a conventional OBD sensor (typically $100-1000 \mathrm{fm} / \sqrt{\mathrm{Hz}}$ in air). Since the bandwidth of the FM detector $\left(B_{\mathrm{FM}}\right)$ is usually less than $1 \mathrm{kHz}$, the deflection noise components which induce the frequency noises should be in the frequency range from $f_{0}-1 \mathrm{kHz}$ to $f_{0}+1 \mathrm{kHz}$. In this frequency region, the experimentally measured values are nearly the same as the theoretically calculated values in both air and liquid environments. Namely, we can obtain the $Q$ limited noise performance in both environments.

\section{B. Frequency noise in FM-AFM}

The deflection noises arising from the deflection sensor and the thermal Brownian motion result in the frequency noise in FM-AFM. The spectral densities of the frequency noise $\left(n_{f}\right)$ is given by

$$
\begin{aligned}
& n_{f}=\sqrt{n_{f s}^{2}+n_{f B}^{2}}, \\
& n_{f s}=\sqrt{2} \frac{n_{z s}}{A} f_{m},
\end{aligned}
$$


(a)

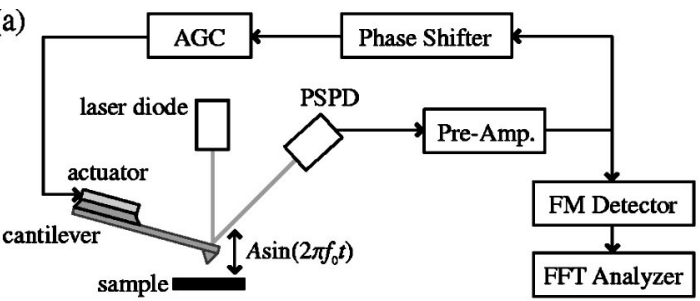

(b)

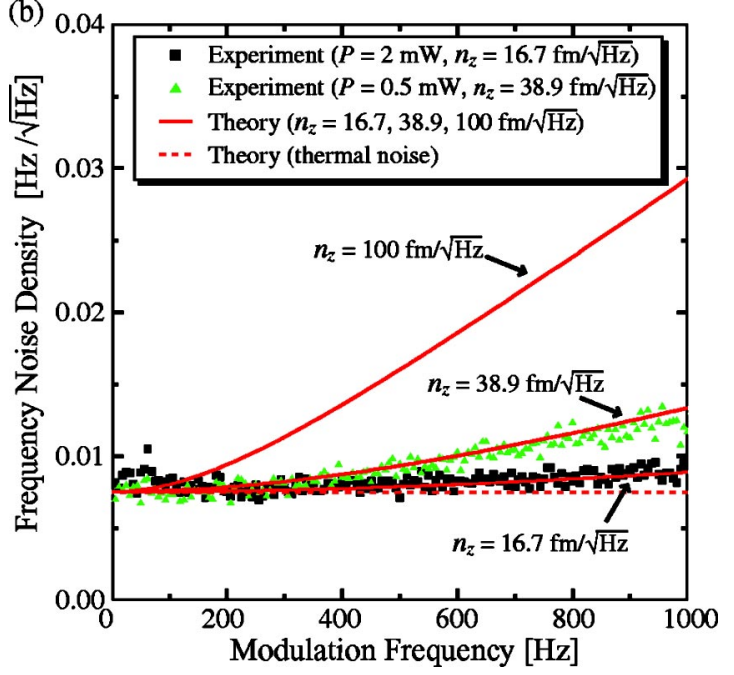

FIG. 9. (Color online) (a) Experimental setup used for the measurement of the frequency noise characteristic of our FM-AFM. (b) Frequency spectra of the frequency shift signal measured with the setup shown in (a) $(A=5 \mathrm{~nm}$, $\left.k=33 \mathrm{~N} / \mathrm{m}, Q=8089, f_{0}=291 \mathrm{kHz}, B_{\mathrm{FM}}=1 \mathrm{kHz}\right)$. The vacuum chamber was pumped with a rotary pump. The vacuum pressure was about $6 \mathrm{~Pa}$. A Si cantilever with Al backside coating (Nanosensors: NCHR) was used. The cantilever was oscillated in constant amplitude mode.

$$
n_{f B}=\sqrt{\frac{k_{B} T f_{0}}{\pi k Q A^{2}}} .
$$

$n_{f s}$ and $n_{f B}$ are the spectral densities of the frequency noises arising from the sensor noise and the thermal Brownian motion, respectively. $f_{m}$ and $A$ denote the modulation frequency of the cantilever vibration frequency $\left(f-f_{0}\right)$ and the vibration amplitude of the cantilever, respectively. These equations show that $n_{f s}$ linearly increases with increasing $f_{m}$ while $n_{f B}$ remains constant. The slope of the linear increase of $n_{f s}$ is proportional to $n_{z}$. Accordingly, the reduction of deflection noise is particularly advantageous for frequency measurements at high modulation frequencies. For example, highspeed FM-AFM imaging requires frequency measurements

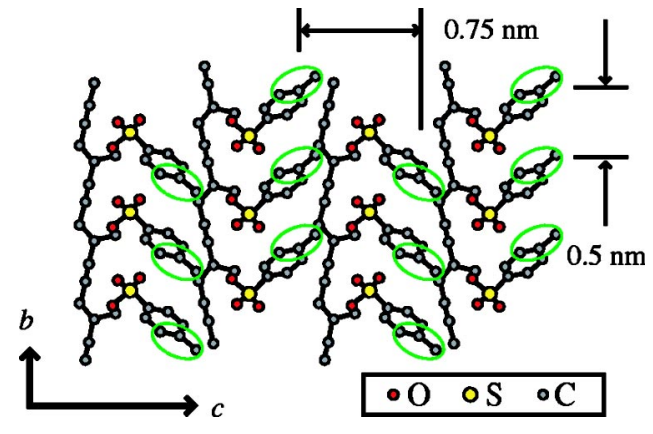

FIG. 10. (Color online) The crystal structure of the $b c$-plane of poly-PTS. The lattice constants of the crystal are $a=1.493 \mathrm{~nm}, b=0.4910 \mathrm{~nm}$, and $c$ $=1.4936 \mathrm{~nm}$. In the $b c$-plane, one side of the PTS side groups is located under the other side. Thus, they are omitted in this figure for clarity. Hydrogen atoms are also omitted to avoid complexity.

at high modulation frequencies. Surface property measurements using frequency modulation technique such as Kelvinprobe force microscopy (KFM) (Ref. 24) and scanning capacitance force microscopy (SCFM) (Ref. 25) require frequency measurements at a modulation frequency of higher than $1 \mathrm{kHz}$.

Figure 9(a) shows the setup used for the measurement of the frequency noise characteristic of our FM-AFM. The cantilever was oscillated with a self-oscillation circuit such that its oscillation amplitude was kept constant $(A=5 \mathrm{~nm})$. The deflection signal from the deflection sensor was fed into the FM detector. Then, the spectral noise densities contained in the output signal of the FM detector was measured with an FFT analyzer (Advantest: R9211B). The measurement was performed in a moderate vacuum environment.

Figure 9(b) shows frequency spectra of the frequency shift signal measured with the setup shown in Fig. 9(a). The deflection noise densities measured with laser powers of $2 \mathrm{~mW}$ and $0.5 \mathrm{~mW}$ were $16.7 \mathrm{fm} / \sqrt{\mathrm{Hz}}$ and $38.9 \mathrm{fm} / \sqrt{\mathrm{Hz}}$, respectively. These values were estimated from the frequency spectra of the thermal Brownian motion. The solid squares and the solid triangles shown in the figure show frequency noise densities measured with laser powers of $2 \mathrm{~mW}$ and $0.5 \mathrm{~mW}$, respectively. The solid lines show frequency noises calculated with Eqs. (10)-(12) for the deflection noise densities of 16.7, 38.9, and $100 \mathrm{fm} / \sqrt{\mathrm{Hz}}$. For both laser powers, the experimental results show good agreement with the theoretical plots.

With a laser power of $2 \mathrm{~mW}, n_{f}$ shows almost constant
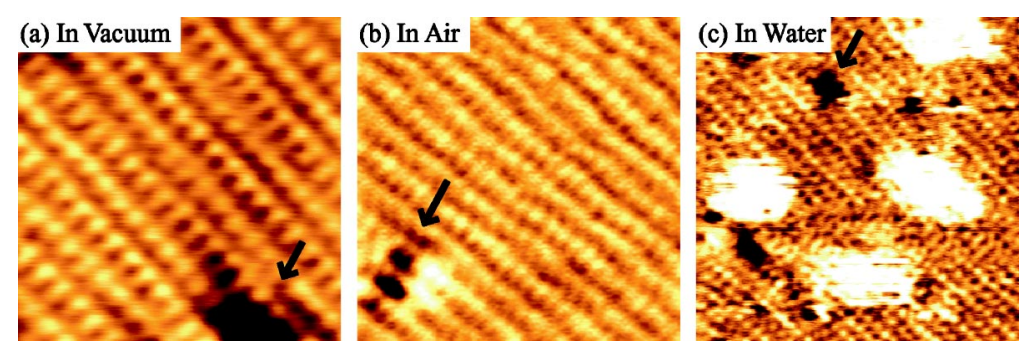

FIG. 11. (Color online) FM-AFM images of the poly-PTS single crystal. (a) In moderate vacuum $(7 \mathrm{~nm} \times 7 \mathrm{~nm}, \Delta f=-70 \mathrm{~Hz}, A=8 \mathrm{~nm}, Q=8089, P=6$ Pa, tip velocity: $560 \mathrm{~nm} / \mathrm{s})$. (b) In air $(10 \mathrm{~nm} \times 10 \mathrm{~nm}, \Delta f=-50 \mathrm{~Hz}, A=4.8 \mathrm{~nm}, Q=241$, tip velocity: $671 \mathrm{~nm} / \mathrm{s})$. (c) In water $(15 \mathrm{~nm} \times 15 \mathrm{~nm}, \Delta f=+200 \mathrm{~Hz}$, $A=0.26 \mathrm{~nm}, Q=27$, tip velocity: $420 \mathrm{~nm} / \mathrm{s})$. A Si cantilever without backside coating (Nanosensors: $\mathrm{NCH})$ was used $(k=42 \mathrm{~N} / \mathrm{m})$. The cantilever was oscillated in constant amplitude mode. 
value with respect to $f_{m}$. The value of $n_{f}$ is nearly the same as the thermal noise limit $\left(n_{f B}\right)$ indicated by the dotted line in Fig. 9(b). This reveals that our low noise deflection sensor makes it possible to obtain $Q$ limited noise performance even with a high $Q$ factor in vacuum and an FM bandwidth of $1 \mathrm{kHz}$. When the laser power was deliberately reduced to $0.5 \mathrm{~mW}, n_{z}$ increased to $38.9 \mathrm{fm} / \sqrt{\mathrm{Hz}}$. In this case, $n_{f}$ shows linear dependence at the modulation frequencies higher than $200 \mathrm{~Hz}$ due to the contribution from the sensor noise. For conventional deflection sensors, $n_{z}$ is larger than $100 \mathrm{fm} / \sqrt{\mathrm{Hz}}$. Thus, the linear dependence becomes more evident as indicated by the theoretical plot for $n_{z}$ of $100 \mathrm{fm} / \sqrt{\mathrm{Hz}}$. In this case, the noise performance of FMAFM is not limited by the thermal Brownian noise but by the sensor noise. Therefore, low noise deflection sensor is essential for obtaining $Q$ limited noise performance.

\section{FM-AFM imaging in various environments}

Using our low noise deflection sensor, we performed FM-AFM imaging in various environments. ${ }^{18,19}$ The sample used here was a single crystal of polydiacetylene, 2,4hexadine-1,6-diol bis(p-toluene sulfonate), which is hereafter referred to as "poly-PTS." A clean surface of the $b c$-plane of poly-PTS was prepared by cleaving it in air. Figure 10 shows the crystal structure of the $b c$-plane of poly-PTS. ${ }^{26}$ In the $b c$-plane, one side of the $p$-toluene sulfonate (PTS) side groups attached to the polydiacetylene main chain protrudes from the molecular $b c$-plane while the other side of the PTS side groups and the main chains are located under the upper PTS side groups. Thus, the topmost carbon atoms indicated by the circles in Fig. 10 should appear as bright protrusions in AFM images of the $b c$-plane. The upper PTS side groups are aligned along the $b$-axis with a separation of $0.491 \mathrm{~nm}$, forming the stripe structures with a period of $0.75 \mathrm{~nm}$ in the $c$-axis direction.

Figures 11(a)-11(c) show FM-AFM images taken in moderate vacuum, air, and water, respectively. All the images show individual PTS side groups separated by about $0.5 \mathrm{~nm}$. In addition, some structural defects are imaged simultaneously with the molecular-scale features as indicated by the black arrows. These results demonstrate that our FM-AFM can achieve true molecular-resolution in every environment. It was possible to obtain molecular resolution with a fast scanning speed. For example, the image shown in Fig. 11(b) was taken with a scanning speed of 33.6 line $/ \mathrm{s}(671 \mathrm{~nm} / \mathrm{s})$ while typical scanning speed in UHV-FM-AFM is 1-3 line/s. Thus, the imaging speed is nearly ten times as fast as those used in conventional FM-AFM. This is because the frequency noises at high modulation frequencies in our FM-AFM are much smaller than that in conventional FMAFM.

The frequency noise in liquid is much larger than those in vacuum or in air due to the low $Q$ factor. In this experiment, we used very small oscillation amplitude of $0.26 \mathrm{~nm}$ for enhancing the force sensitivity of the frequency shift signal to the short-range interaction forces. The low noise characteristic of our deflection sensor was essential for the stable cantilever self-oscillation with such a small oscillation amplitude. The detailed experimental conditions and the imaging mechanism of the liquid-environment FM-AFM are discussed elsewhere. ${ }^{19}$

\section{ACKNOWLEDGMENTS}

The authors thank S. Okada and H. Nakanishi for providing poly-PTS single crystals. This work was supported by a Grant-in-Aid and a Leading Project on Nanotechnology and Materials from the Ministry of Education, Culture, Sports, Science and Technology of Japan, and the 21st Century Center of Excellence Program, Kyoto University.

${ }^{1}$ T. R. Albrecht, P. Grütter, D. Horne, and D. Ruger, J. Appl. Phys. 69, 668 (1991).

${ }^{2}$ F. J. Giessibl, Science 267, 68 (1995).

${ }^{3}$ S. Kitamura and M. Iwatsuki, Jpn. J. Appl. Phys., Part 2 34, L1086 (1995).

${ }^{4}$ S. Orisaka, T. Minobe, T. Uchihashi, Y. Sugawara, and S. Morita, Appl. Surf. Sci. 140, 243 (1999).

${ }^{5}$ C. Loppacher, M. Bammerlin, M. Guggisberg, F. Battiston, R. Bennewitz, S. Rast, A. Baratoff, E. Meyer, and H.-J. Güntherodt, Appl. Surf. Sci. 140, 287 (1999).

${ }^{6}$ M. Bammerlin, R. Lüthi, E. Meyer, A. Baratoff, J. Lü, M. Guggisberg, C. Gerber, L. Howald, and H.-J. Güntherodt, Probe Microsc. 1, 3 (1997).

${ }^{7}$ K. Fukui, H. Onishi, and Y. Iwasawa, Chem. Phys. Lett. 280, 296 (1997).

${ }^{8}$ G. Binnig, C. F. Quate, and C. Gerber, Phys. Rev. Lett. 56, 930 (1986).

${ }^{9}$ Y. Martin, C. C. Williams, and H. K. Wickramasinghe, J. Appl. Phys. 61, 4723 (1987).

${ }^{10}$ G. M. McClelland, R. Erlandsson, and S. Chiang, Review of Progress in Quantitative Non-Destructive Evaluation (Plenum, New York, 1988), Vol. 6B, pp. 1307-1314.

${ }^{11}$ G. Meyer and N. M. Amer, Appl. Phys. Lett. 53, 1045 (1988).

${ }^{12}$ C. Schönenberger and S. F. Alvarado, Rev. Sci. Instrum. 60, 3131 (1989).

${ }^{13}$ G. Neubauer, S. R. Cohen, G. M. McClelland, D. Horne, and C. M. Mate, Rev. Sci. Instrum. 61, 2296 (1990).

${ }^{14} \mathrm{M}$. Tortonese, H. Yamada, R. C. Barrett, and C. F. Quate, The Proceedings of Transducers ' 91 (IEEE, Pennington, NJ, 1991), pp. 448-451, publication No. 91 CH2817-5.

${ }^{15}$ T. Itoh and T. Suga, Nanotechnology 4, 218 (1993).

${ }^{16}$ D. Rugar, H. J. Mamin, R. Erlandsson, J. E. Stern, and B. D. Terris, Rev. Sci. Instrum. 59, 2337 (1988).

${ }^{17}$ C. A. J. Putman, B. G. D. Grooth, N. F. V. Hulst, and J. Greve, J. Appl. Phys. 72, 6 (1992).

${ }^{18}$ T. Fukuma, T. Ichii, K. Kobayashi, H. Yamada, and K. Matsushige, Appl. Phys. Lett. 86, 034103 (2005).

${ }^{19}$ T. Fukuma, K. Kobayashi, K. Matsushige, and H. Yamada, Appl. Phys. Lett. (submitted).

${ }^{20}$ D. Sarid, Scanning Force Microscopy with Applications to Electric, Magnetic and Atomic Forces (Oxford University Press, Oxford 1994).

${ }^{21}$ M. Ojima, A. Arimoto, N. Chinone, T. Gotoh, and K. Aiki, Appl. Opt. 25, 1404 (1986)

${ }^{22}$ A. Arimoto, M. Ojima, N. Chinone, A. Oishi, T. Gotoh, and N. Ohnuki, Appl. Opt. 25, 1398 (1986).

${ }^{23}$ K. Kobayashi, H. Yamada, H. Itoh, T. Horiuchi, and K. Matsushige, Rev. Sci. Instrum. 72, 4383 (2001).

${ }^{24}$ S. Kitamura and M. Iwatsuki, Appl. Phys. Lett. 72, 3154 (1998).

${ }^{25}$ K. Kobayashi, H. Yamada, and K. Matsushige, Appl. Phys. Lett. 81, 2629 (2002).

${ }^{26}$ V. D. Kobelt and E. F. Paulus, Acta Crystallogr., Sect. B: Struct. Crystallogr. Cryst. Chem. 30, 232 (1974). 